\title{
Intelligent Decision System of Higher Educational Resource Data Under Artificial Intelligence Technology
}

\author{
https://doi.org/10.3991/ijet.v16i05.20305 \\ Junhong Ma \\ Xi'an International University, Xi'an, China \\ 2340869 @qq. com
}

\begin{abstract}
It aims to apply the neural network algorithm to the mining of educational resource data and provide new ideas for the intelligent development of teaching evaluation. The Apriori algorithm is modified with the decision tree based on the research of existing university teaching evaluation system. The modified Apriori algorithm is applied to analyze the correlations of the teaching evaluation results to the teacher's age, gender, professional title, and academic qualification. The back propagation (BP) neural network model is improved as the DEA-BP based on the differential evolution algorithm (DEA). The DEA-BP model is applied to the prediction of teaching evaluation results for analysis. The results show that the execution time of the modified Apriori algorithm is significantly better than that of other models. In addition, the teacher's age (40 50 years old or 50 - 60 years old), gender (female), professional title (senior or deputy senior), and academic qualifications (undergraduate or master) have reliable correlation with the teaching evaluation results (excellent). When the DEA-BP algorithm is adopted to predict the teaching evaluation results, the average absolute error $(1.05 \%)$ and the relative accuracy rate $(95.44 \%)$ between its prediction value and the true value are optimal. Therefore, the Apriori algorithm and DEA-BP algorithm can intelligently extract the potential laws and knowledge in the teaching evaluation data, and provide support for teaching evaluation decisions. Thus, it exerts the role of promotion in the mining of educational resource data in universities and the intelligent development of decision-making systems.
\end{abstract}

Keywords-Decision tree; Apriori algorithm; differential evolution algorithm; BP neural network; teaching evaluation

\section{Introduction}

The popularization of higher education is also accelerating day by day with the continuous deepening of education reform, so the status of teaching level evaluation and its role in education reform have become increasingly important [1]. The indicators for teaching evaluation are vague and diverse, and the filling is used to obtain the levels of "excellent", "good", "qualified", and "unqualified" in practice [2]. Teaching evaluation can play an important role in promoting the development of the college and ensuring the quality of talent training in college. At present, the educational re- 
source data is massive, multi-dimensional, and dynamic. How to mine the connections among internal factors from these data, and then transfer the extracted knowledge to the decision support system [3]? The main forms of decision support systems are databases, expert systems, and artificial intelligence systems combining the database and data mining [4]. The advantage of data mining technology is that it can realize indepth analysis through computer technology with the existing information. Thus, it can greatly reduce the use of manpower and material resources, and has important significance for college education managers to make important decisions [5]. The algorithms commonly used in data mining currently include the decision trees, association rules, and random forests [6]. Applying the neural network algorithms in the mining of educational resource data can provide new ideas for the intelligent development of teaching evaluation.

Therefore, the teaching evaluation intelligent decision-making system is optimized with the improved data mining algorithm based on the existing teaching evaluation systems of Xi'an International University. In addition, the results of this study are of great significance for promoting the intelligent development of teaching evaluation.

\section{$2 \quad$ Methodology}

\subsection{Basic framework of decision support system based on the education data mining}

Data mining technology refers to obtaining the hidden, potential, and advanced information from a large amount of data in a database or data warehouse. The combination of data mining, database, and online analytical processing technology can enable the system more comprehensive [7]. The basic structure of the decision support system based on the educational resource data mining proposed based on the above theory is shown in Figure 1. It indicates that the system covers the database technology, online analytical processing technology, and data mining technology.

\subsection{Improvement on fast association rules based on the decision tree}

The decision tree [8] is to classify the educational resource data. The classified data is mined with the modified Apriori algorithm, which is compared with other algorithms to prove its effectiveness. When a decision tree is constructed, it is necessary to reduce the disorder in the new child nodes that appear after splitting, so the quality of the node splitting has to be introduced by using the introduced indicators. In addition, the information gain (IG) and Gini index are incorporated into the decision tree in this study. As an important algorithm in the classification algorithm under the decision tree, the Iterative Dichotomiser 3 (ID3) is to select the attribute of each hierarchical node of the decision tree. It is necessary to use IG to select the attribute, and finally to achieve the obtained. test of each non-leaf node. The largest category information recorded by the test can be obtained. The attribute with the largest IG can be obtained based on the comparison of the IG sizes of different attributes. 


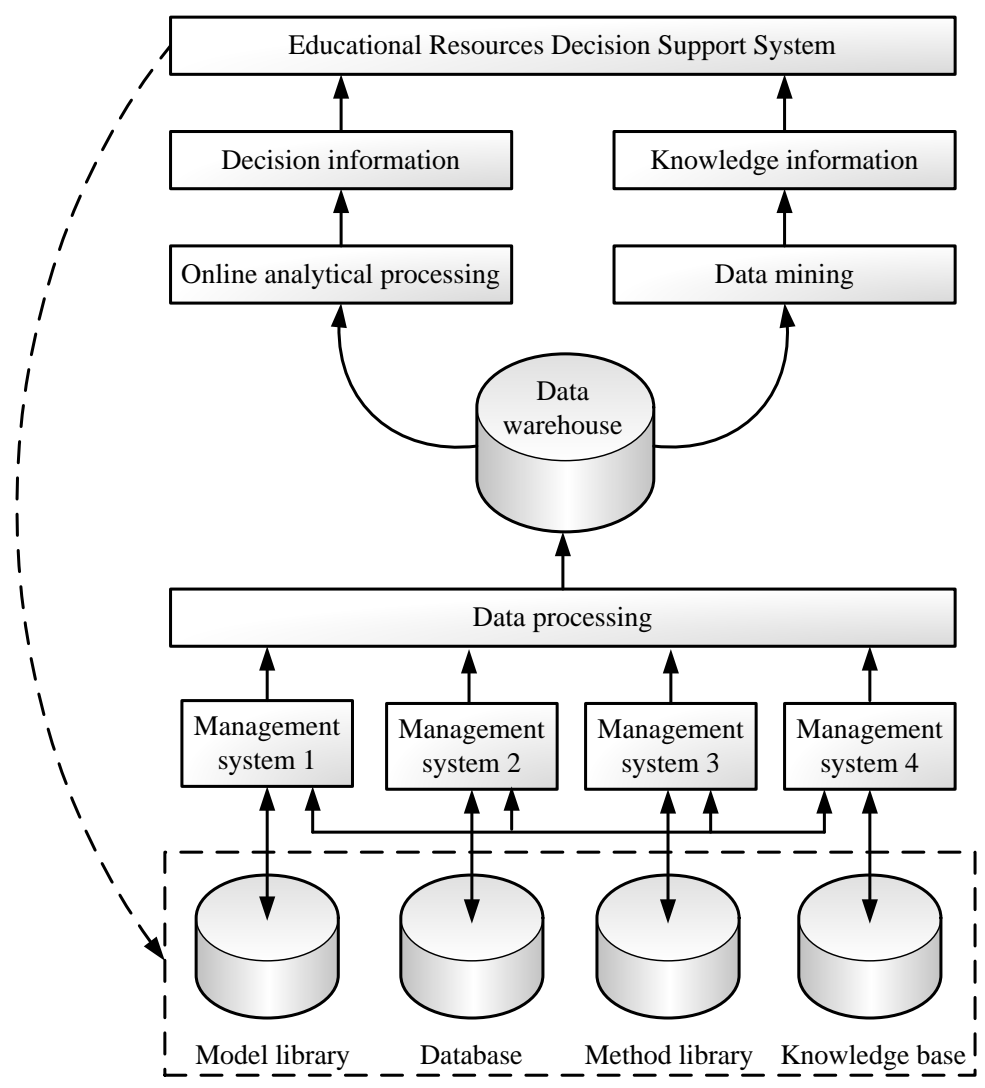

Fig. 1. Basic structure of the decision support system based on the educational resource data mining

It is assumed that the set of data samples is A and the category label is $n$, then $n$ different categories are $\mathrm{Ci}$. If $\mathrm{Ai}$ is the number of samples in $\mathrm{Ci}$, then the expected value of information for sample classification can be expressed as equation (1):

$$
I\left(A_{1}, A_{2}, \cdots, A_{n}\right)=-\sum(i=1,2, \cdots, n) p_{i} \log _{2}\left(p_{i}\right)
$$

Equation (1) reveals that the total entropy of the system is the weighted average of the information amount of different categories of data, and $p_{i}=A_{i} / A$ (that is, the probability that any sample belongs to the category $\mathrm{Ci}$ ).

It is supposed that attribute $\mathrm{S}$ contains $\mathrm{m}$ different values $(\mathrm{s} 1, \mathrm{~s} 2, \ldots, \mathrm{sm})$, so A can be divided into different subsets (A1, A2, .., Am) with the attribute $\mathrm{S}$, where the sample of subset $\mathrm{Ai}$ is $\mathrm{Sj}(\mathrm{j}=1,2, \ldots, \mathrm{m})$ with the same value on the attribute $\mathrm{S}$. If $\mathrm{Aij}$ is the number of samples in the category $\mathrm{Ci}$ of the data in the subset $\mathrm{Aj}$, the expected value of information for dividing the data set $\mathrm{S}$ into subsets is given as below:

$$
E(S)=\frac{\sum_{j=1}^{m}\left(a_{1 j}+a_{2 j}+\cdots+a_{n j}\right)}{a \cdot I\left(A_{1}, A_{2}, \cdots, A_{n}\right)}
$$


The smaller the expected value of information, the higher the purity of the subset. If a certain subset a is given, the expected value of its information can be calculated with below equation:

$$
I\left(A_{1 j}, A_{2 j}, \cdots, A_{n j}\right)=-\sum_{i=1}^{n} p_{i j} \log _{2}\left(p_{i j}\right)
$$

Then, the obtainable IG of the subset in the attribute $\mathrm{S}$ can be expressed as below:

$$
I G(S)=I\left(A_{1}, A_{2}, \cdots, A_{n}\right)-E(S)
$$

Then, the On-Line Analysis Processing (OLPA) [9] is used for modification of the Apriori algorithm, the central idea of which is given as follows. All items in the data set whose number of items are less than $\mathrm{k}$ have no impact on the frequent data set that produces $\mathrm{k}$ items, it has to find a set of $\mathrm{k}$ length in the index item when looking for a set of frequent $\mathrm{k}$ items; the candidate data is determined by the data from the database, and then the data set Lk of the frequent item $\mathrm{k}$ can be determined. In this way, the scanning efficiency of the algorithm can be improved. In order to obtain the specific location of the data transferred into the memory, the index table is introduced to search for set of frequent item $\mathrm{k}$, and is constructed with a conventional method. The length of the data combination is set to Len. The basic process for modification of the Apriori algorithm is shown in Figure 2.

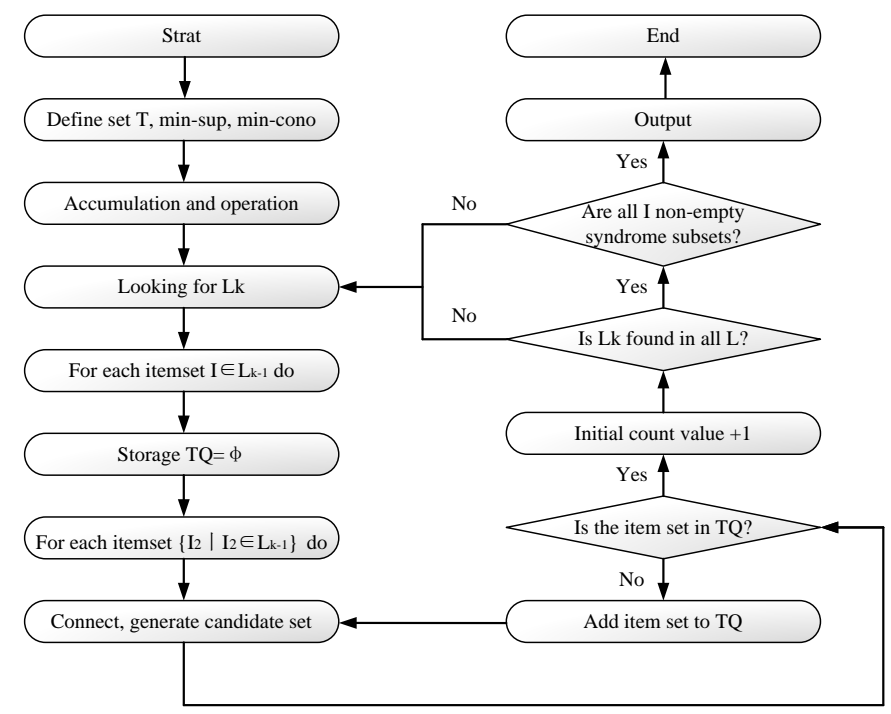

Fig. 2. Modification process of Apriori algorithm based on OLPA

A fast Apriori algorithm is proposed based on the Apriori algorithm modified with OLPA and combining with the ID3, and the implementation flows of the algorithm are shown in Figure 3 as below. 


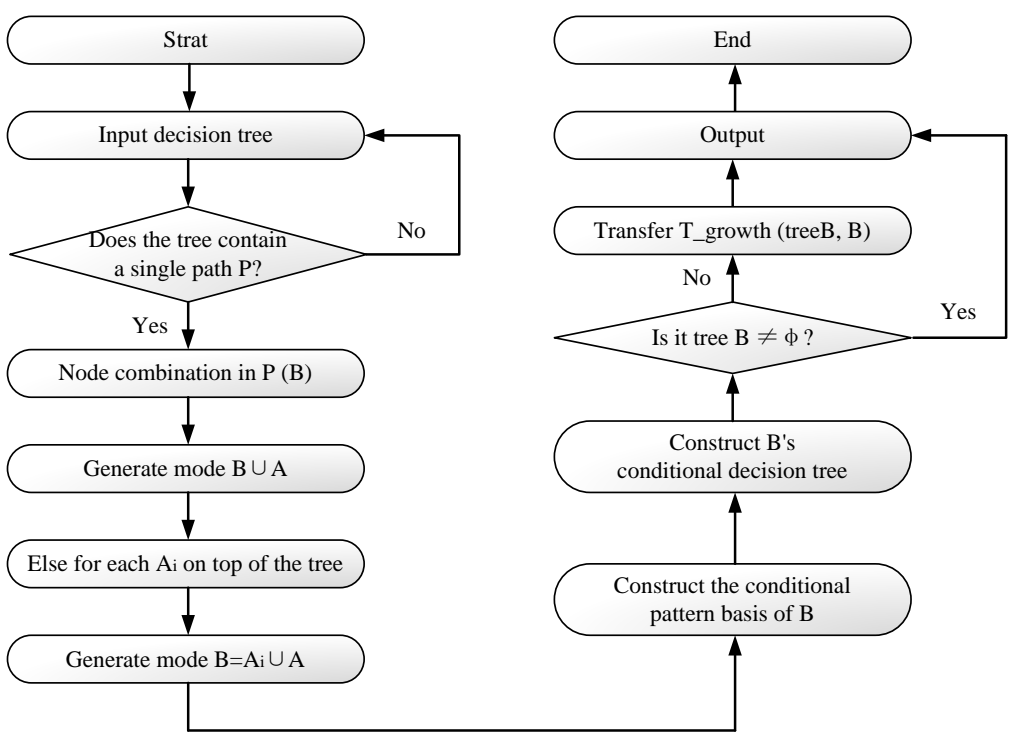

Fig. 3. Implementation flows of fast Apriori algorithm

\subsection{Construction of BP neural network model based on differential evolution algorithms}

The typical DEA is similar to genetic algorithms (GA), and also involves three kinds of operations: selection, crossover, and mutation [10]. If the expression of an individual is xi, $\mathrm{G}$, where $\mathrm{G}$ is an algebra. In the operation of mutation, a new mutant individual Ui, G can be generated for each individual xi, G with the following operations, which can be used as a random variable:

$$
U_{i, G}=x_{r_{1}, G}+F\left(x_{r_{2}, G}-x_{r_{3}, G}\right)
$$

In the above equation, $F \in[0,2]$.

In the operation of crossover, a random variable Randomi is firstly generated, and the crossover between the target individual $\mathrm{xi}, \mathrm{G}$ and the mutated individual $\mathrm{Ui}, \mathrm{G}$ is performed to generate a test individual Vi, G. During generation of the test individual, it is necessary to perform individual evolution and ensure that at least one of the randomly selected test individuals $\mathrm{Vi}, \mathrm{G}$ can contribute to the $\mathrm{Ui}, \mathrm{G}$. The process of crossover is given as below:

$$
v_{j i, \mathrm{G}}=\left\{\begin{array}{l}
u_{j i, G}, \quad \text { Rand }_{j} \leq C R \quad \text { or } j=\text { Random }_{i} \\
x_{j i, G}, \quad \text { Rand }_{j}>C R \text { and } j \neq \text { Random }_{i}
\end{array}\right.
$$

In the above equation, Randj is a random real number evenly distributed in the range $[0,1], \mathrm{CR}$ is a crossover factor and is in the range of $[0,1]$, and Randomi is a random integer in the range of $(1,2, \ldots, \mathrm{m})$. 
The greedy selection strategy can be used for selection. It is assumed that the newly generated descendant vi, G+1 has a better fitness than xi, G, then the descendant vi, $\mathrm{G}+1$ can enter the next generation, otherwise the descendant vi, $\mathrm{G}+1$ is retained. The process of the selection is shown as below:

$$
x_{i, G+1}=\left\{\begin{array}{lll}
v_{i, G}, & \text { if } & \text { fit }\left(v_{i, G}\right)<f i t\left(x_{i, G}\right) \\
x_{i, G}, & \text { if } & f i t\left(v_{i, G}\right) \geq f i t\left(x_{i, G}\right)
\end{array}\right.
$$

The implementation flow of DEA is shown in Figure 4. The parameters involved in DEA include the mutation factor, population size, crossover probability factor, dimension, maximum number of iterations, and termination conditions.

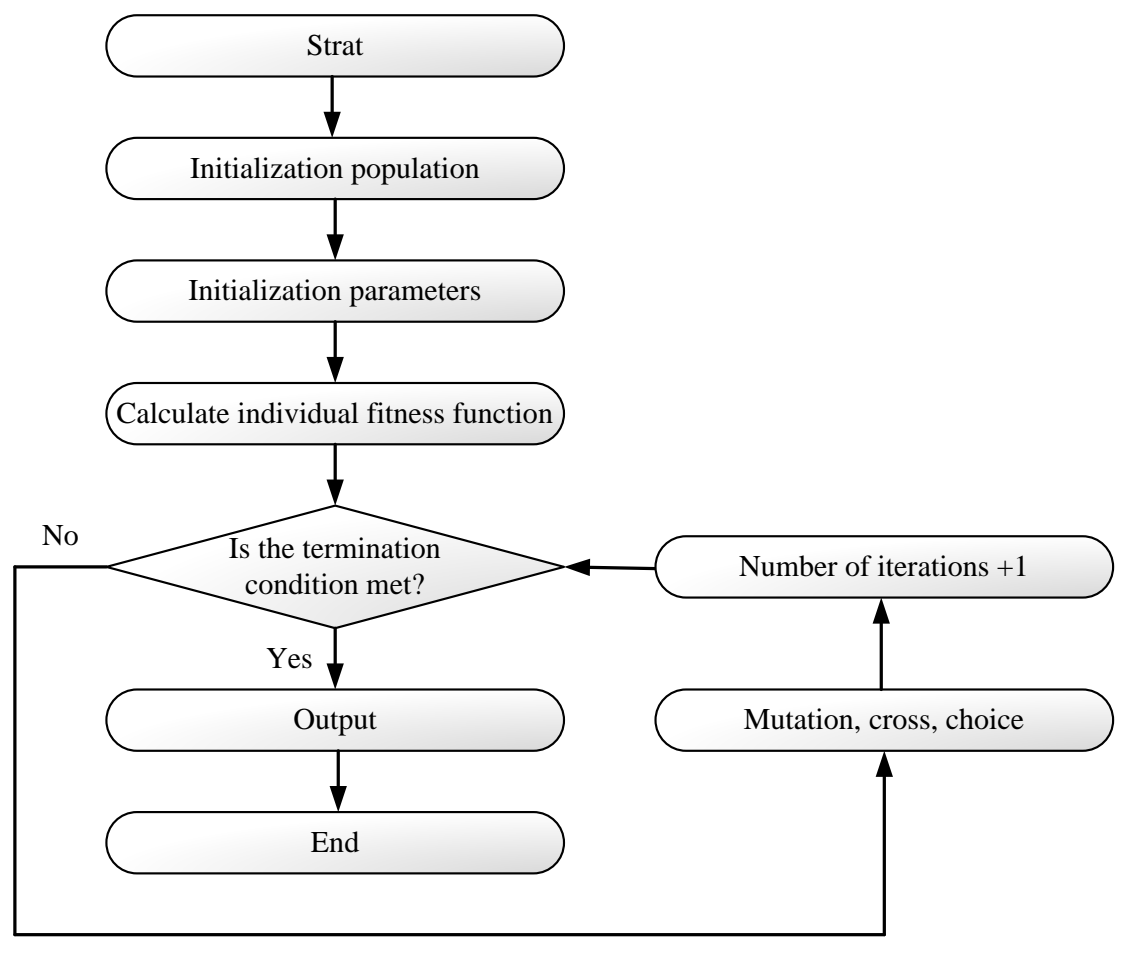

Fig. 4. Calculation flows of DEA

When the population size or crossover probability factor is too large, the complexity of the algorithm will be increased. Small population size or crossover probability factor will cause the algorithm to premature, so that it falls into the local optimal solution. Thus, the population size has to be greater than 4 , and the crossover probability factor has to be determined according to the actual situation. If the variation factor is too large, the local optimal solution also can be resulted in; while if it is too small, the difference between populations will be small. In addition, the number of iterations in this study is set to 10 , and the termination condition is set to 10-6. 
BP neural network model is mainly composed of input layer, hidden layer, and output layer. The calculation process of the BP neural network algorithm is shown in Figure 5. The Adaptation moment estimation (Adam) algorithm [11] is undertaken as the optimization algorithm in the construction of the BP neural network to overcome the local minimum value caused by the original Stochastic gradient descent (SGD) algorithm [12].

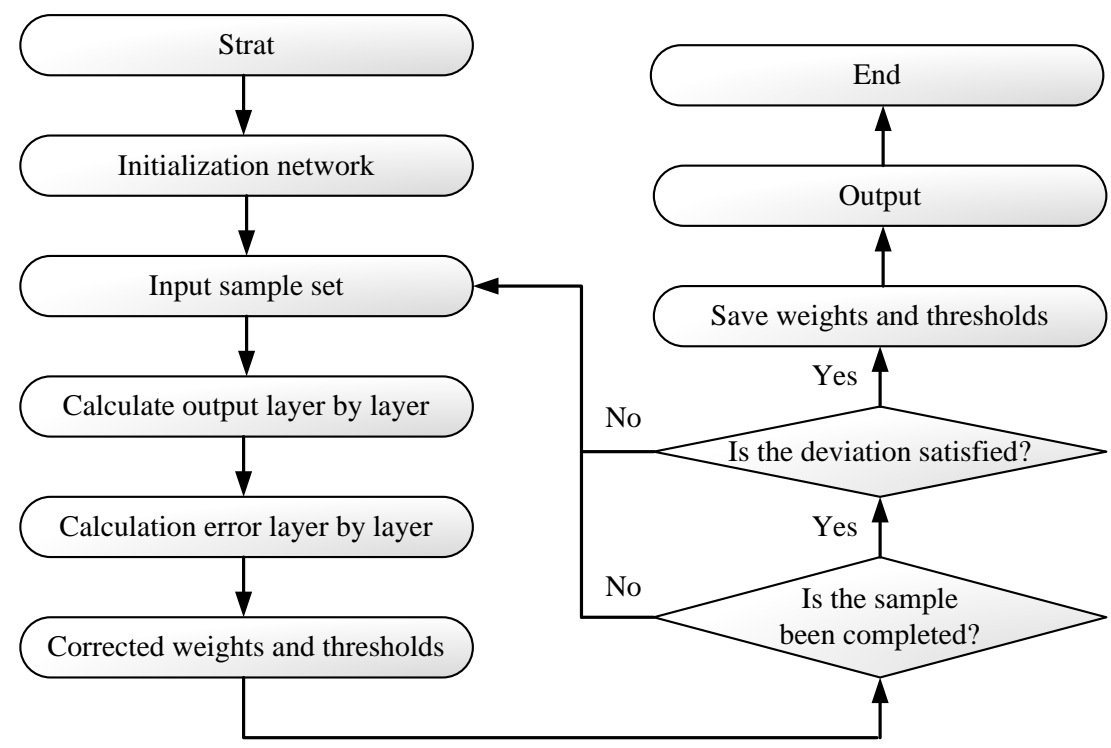

Fig. 5. Calculation process of BP neural network

When it is optimized with Adam, the mathematical expressions for gradient calculation can be expressed as below:

$$
\begin{aligned}
o_{k} & =\beta_{1} o_{k-1}+\left(1-\beta_{1}\right) g_{k} \\
t_{k} & =\beta_{2} t_{k-1}+\left(1-\beta_{1}\right) g_{k}
\end{aligned}
$$

In the above equation, ok is the first-order matrix estimate of the gradient, tk is the second-order matrix estimate of the gradient, and the initial values of ok and tk are both 0 vectors.

Then the values of ok and tk after the deviation correction can be calculated by equation (10) and equation (11):

$$
\begin{aligned}
& \overline{o_{k}}=\frac{o_{k}}{1-\beta_{1}^{k}} \\
& \overline{t_{k}}=\frac{t_{k}}{1-\beta_{2}^{k}}
\end{aligned}
$$


Based on the corrected ok and tk, the expression of Adam algorithm can be updated as:

$$
\theta_{k}=\theta_{k-1}-\frac{\eta}{\sqrt{\overline{t_{k}}+\varepsilon}} \overline{o_{k}}
$$

In the above equations (10), (11), and (12), $\eta=0.001, \beta 1=0.9, \beta 2=0.99$, and $\varepsilon=10-8$.

In this study, DEA is applied to construct the BP neural network model, and the operation of Dropout is introduced during the training of the BP neural network [13]. The implementation process of the DEA-BP neural network model is shown in Figure 6.

The DEA-BP neural network algorithm is calculated based on the process shown in Figure 6. Firstly, the populations and parameters are initialized. The real vector can be used as a single sample of the population by using the following equation:

$$
S=\left\{\omega_{1}, \theta_{1}, \omega_{2}, \theta_{2}, \omega_{3}, \theta_{3}\right\}
$$

In the above equation, $\omega$ is the weight value, $\theta$ is the threshold, 1 represents the input layer and hidden layer, 2 represents the first and the second layer of hidden layer, and 3 represents the hidden layer and output layer.

Secondly, the individual map is undertaken as the weight value and threshold of different nodes in the BP neural network. What's more, the error value is obtained after the forward propagation of the network is completed and then undertaken as the fitness function of the individual. The mutation, crossover, and selection have to be taken if the fitness function does not meet the termination condition. It has to return to the previous step with the next generation, or otherwise start the next step. The optimal initial parameters can be obtained later, and the BP neural network is running. When the network reaches the optimal parameter value, the entire training of the model is completed. 

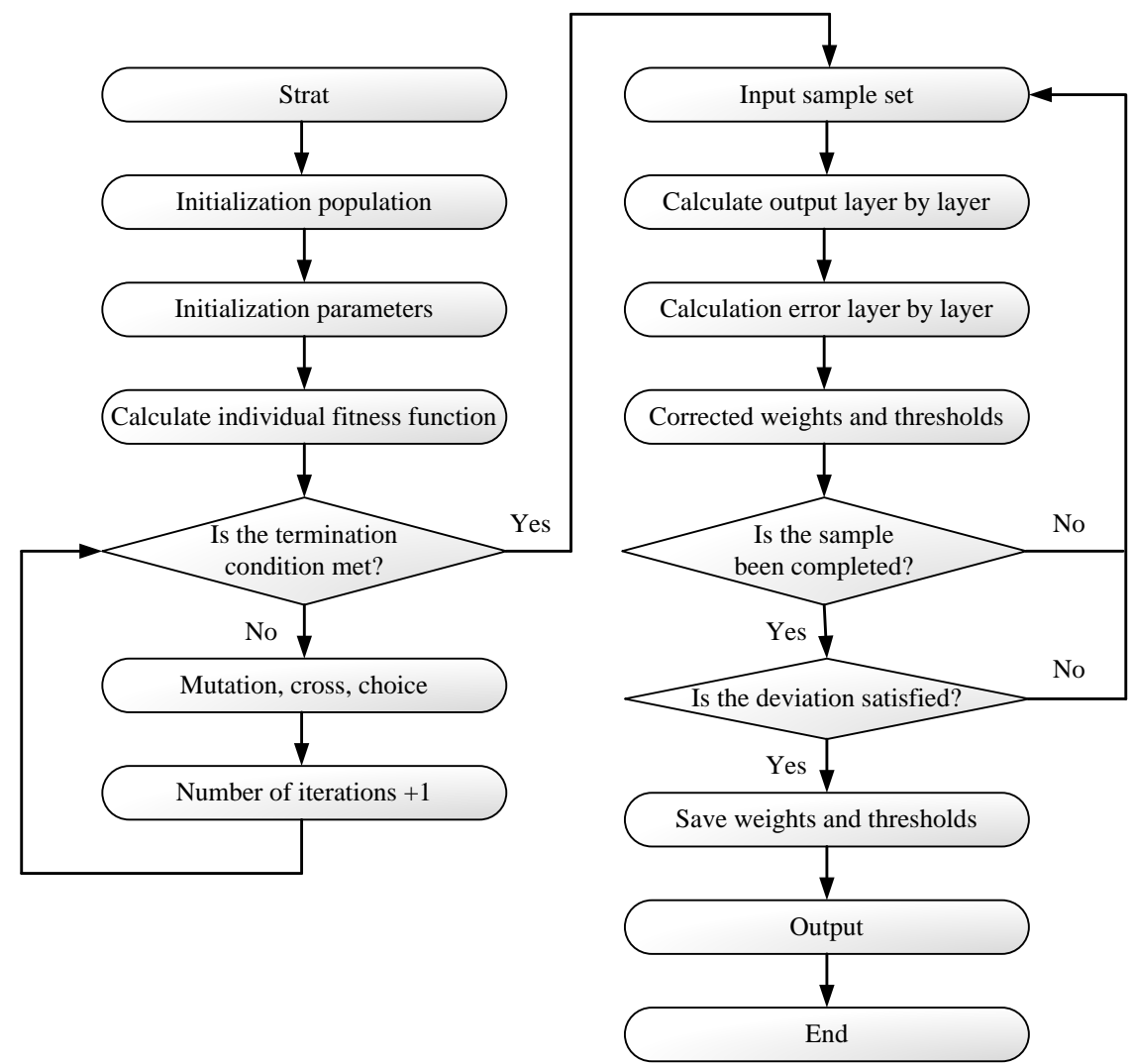

Fig. 6. Implementation process of the DEA-BP neural network model

\subsection{Preprocess of the higher educational resource data}

The teaching evaluation data of students from Xi'an International University in the 2018 - 2019 school year are selected as the research objects in this study. The inconsistent date under the sorted original data are corrected through filling vacant data, deleting abnormal data, and smoothing noise data. The multiple data are stored and combined into one data set for data mining, and then correlation analysis is used to eliminate the redundant data. The equation for correlation analysis is given as below:

$$
r_{x, y}=\frac{\sum(x-\bar{x})(y-\bar{y})}{(n-1) \sigma_{x} \sigma_{y}}
$$

In the above equation, $\mathrm{x}$ and $\mathrm{y}$ are two different types of attributes, $\bar{x}$ and $\bar{y}$ are the mean values of attribute $\mathrm{x}$ and $\mathrm{y}$, and $\sigma_{x}$ and $\sigma_{y}$ are the standard deviations of attribute $\mathrm{x}$ and $\mathrm{y}$, respectively. When $\mathrm{r}>0$, the correlation between attribute $\mathrm{x}$ and $\mathrm{y}$ is positive; when $r=0$, there is no correlation between attribute $x$ and $y$; when $r<0$, the attribute $\mathrm{x}$ has a negative correlation with the attribute $\mathrm{y}$. 
After the calculation using above equation, it is assumed that $\mathrm{x}$ is the age, $\mathrm{y}$ is the score, and $\mathrm{n}$ is 209 , then the calculated $\mathrm{r} \approx 0.259$, indicating that the teacher's age is positively correlated with the score. In order to ensure the data of the input model meet the requirements, data conversion is realized based on the processed data. The title can be classified with following principles: professor and other position with senior professional title are classified as "senior", associate professor and other deputy senior title are classified as "deputy senior", lecturer and other intermediate professional title are classified as "intermediate", and teaching assistant and other primary title are classified as "junior". The age can be divided as < 30, 30 - 40, 40 - 50, 50 60 , and $>60$. The academic qualification can be divided into "excellent" with score of $>4.5$, "good" with score of $4-4.5$, "qualified" with score of $3.5-4$, and "unqualified" with score of $<3$.

Finally, the IG value of each category of data is calculated. The IG value of the teaching score can be calculated with below equation:

$$
I(151,167,2)=-\frac{151}{320} \log _{2} \frac{151}{320}-\frac{167}{320} \log _{2} \frac{167}{320}-\frac{2}{320} \log _{2} \frac{2}{320} \approx 1.019
$$

The information entropy and IG value of different gender in the teaching scoring registration can be calculated with below equations:

$$
\begin{gathered}
E(\text { gender })=\frac{181}{320} I(85,96)+\frac{139}{320} I(66,71,2) \approx 0.989 \\
\text { Gian }(\text { gender })=I(151,167,2)-E(\text { gender })=0.030
\end{gathered}
$$

In the same way, the information entropy and IG value of different ages, titles, and academic qualification can be calculated, which should be $(1.005,0.014),(1.003$, $0.016)$, and $(0.998,0.021)$, respectively.

\subsection{Verification of fast Apriori algorithm based on the decision tree}

Based on the classification data after the pre-processing, the execution time of the constructed algorithm is compared with that of the decision tree, frequent pattern growth [14], and random forest [15]. The comparison results are shown in Figure 7 below. It illustrated that the proposed algorithm has the shortest execution time under different sample sizes. The execution time of different algorithms presents a trend of: decision tree $>$ frequent pattern - growth $>$ random forest $>$ the proposed algorithm. It shows that the proposed algorithm has obvious advantages with the increase of data volume and the improved execution speed, which verifies the practicability and feasibility of the algorithm. 


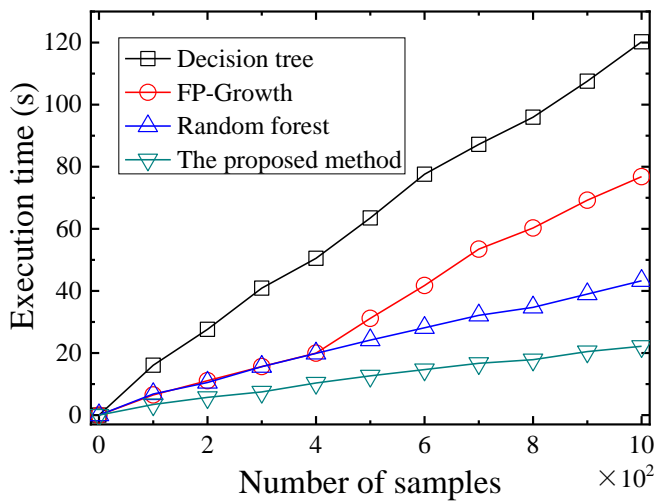

Fig. 7. Simulation comparison on execution time of different algorithms

Firstly, the decision tree is to generate the rules among different projects, and the results are shown in Table 1. It suggests that the teaching evaluation results can be well predicted by the teacher's academic qualification and professional title, but the dependence relationship between the teacher's academic qualification and professional title is not obvious.

Table 1. Original teaching evaluation information

\begin{tabular}{|c|c|c|c|c|}
\hline Path & $\begin{array}{l}\text { Teaching } \\
\text { evaluation } \\
\text { results }\end{array}$ & $\begin{array}{l}\text { Number } \\
\text { (persons) }\end{array}$ & Probability & Description \\
\hline \multirow{3}{*}{ All } & Qualified & 2 & $1.45 \%$ & \multirow{3}{*}{$\begin{array}{l}\text { The probability of being "good" is the highest } \\
\text { without considering other influencing factors. }\end{array}$} \\
\hline & Good & 167 & $51.26 \%$ & \\
\hline & Excellent & 151 & $47.29 \%$ & \\
\hline \multirow{3}{*}{ "Doctor" } & Qualified & 0 & $11.38 \%$ & \multirow{3}{*}{$\begin{array}{l}\text { The probability of academic qualification as } \\
\text { "doctor" and teaching evaluation result as "good" } \\
\text { is the highest. }\end{array}$} \\
\hline & Good & 5 & $58.15 \%$ & \\
\hline & Excellent & 1 & $30.47 \%$ & \\
\hline \multirow{3}{*}{ "Senior" } & Qualified & 0 & $9.22 \%$ & \multirow{3}{*}{$\begin{array}{l}\text { The probability of title as "senior" and teaching } \\
\text { evaluation result as "excellent" is the highest. }\end{array}$} \\
\hline & Good & 2 & $35.39 \%$ & \\
\hline & Excellent & 3 & $55.39 \%$ & \\
\hline \multirow{3}{*}{$\begin{array}{l}\neq \text { "Doctor" } \\
=\text { "Senior" }\end{array}$} & Qualified & 0 & $8.82 \%$ & \multirow{3}{*}{$\begin{array}{l}\text { The probability of academic qualification as "not } \\
\text { doctor" and the teaching evaluation results as } \\
\text { "excellent" is the highest. }\end{array}$} \\
\hline & \begin{tabular}{|l|} 
Good \\
\end{tabular} & 1 & $32.17 \%$ & \\
\hline & Excellent & 4 & $59.01 \%$ & \\
\hline
\end{tabular}

Then, the mining model is used for data mining, and the results are shown in Figure 8 . The overall predictive accuracy rate becomes a linear growth trend when total of the proposed data mining model is $0 \%-50 \%$; the overall predictive accuracy rate remains unchanged status (around 50\%) when the total of the data mining model proposed in this study is $50 \%-70 \%$; and the overall predictive accuracy rate shows a trend of linear growth again when it exceeds $70 \%$. 




Fig. 8. Simulation figure of mining increase of the mining model proposed in this study

The association rules are used for data mining, and the generation rules are used to build the dependency network graph, as illustrated in Figure 9.

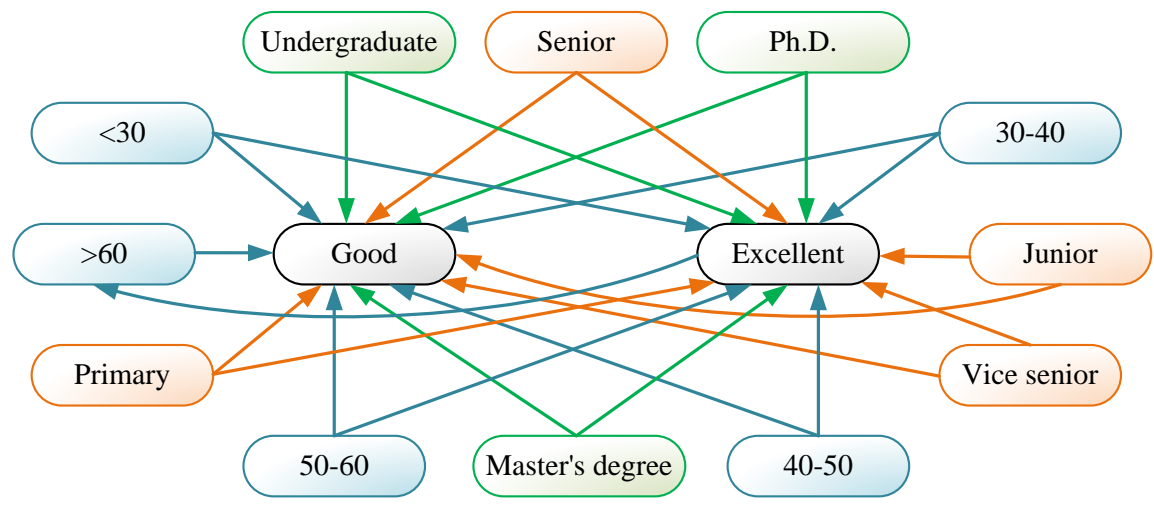

Fig. 9. Network graph of dependency base on the association rules

The associated data of teacher's age, professional title, academic qualification, gender, and teaching evaluation results are mined based on the above results. The results are shown in Table 2. Table 2 discloses that the teaching evaluation results of teachers aged $40-50$ and $50-60$ are better. Teachers with senior and deputy senior professional titles have better teaching evaluation results. The teaching evaluation results of teachers with undergraduate and master in academic qualification are better. The teaching evaluation results of female teachers are better. It means that female teacher with older age, higher professional title, and the academic qualification of undergraduate or master has the better teaching effect, which may because that the 
older teacher has more teaching experiences and the female teacher is more careful $[16,17]$.

Table 2. Mining results of associated data

\begin{tabular}{|l|l|c|c|}
\hline \multicolumn{1}{|c|}{ Item } & \multicolumn{1}{|c|}{ Set } & Degree of support & Confidence coefficient \\
\hline \multirow{5}{*}{ Age } & $<30$, good & $0.66 \%$ & $50.47 \%$ \\
\cline { 2 - 4 } & $30-40$, good & $4.52 \%$ & $55.40 \%$ \\
\cline { 2 - 4 } & $40-50$, excellent & $5.38 \%$ & $52.19 \%$ \\
\cline { 2 - 4 } & $50-60$, excellent & $13.39 \%$ & $68.92 \%$ \\
\cline { 2 - 4 } & $>60$, excellent & $1.47 \%$ & $43.01 \%$ \\
\hline \multirow{4}{*}{$\begin{array}{l}\text { Professional } \\
\text { title }\end{array}$} & Junior, good & $3.38 \%$ & $54.32 \%$ \\
\cline { 2 - 4 } & Intermediate, good & $6.08 \%$ & $55.06 \%$ \\
\cline { 2 - 4 } & Deputy senior, excellent & $11.16 \%$ & $57.34 \%$ \\
\cline { 2 - 4 } & Senior, excellent & $8.97 \%$ & $60.12 \%$ \\
\hline \multirow{3}{*}{ Academic } & Undergraduate, excellent & $9.98 \%$ & $58.11 \%$ \\
\cline { 2 - 4 } & Master, excellent & $8.79 \%$ & $56.34 \%$ \\
\cline { 2 - 4 } & Doctor, good & $18.37 \%$ & $87.14 \%$ \\
\hline \multirow{2}{*}{ Gender } & Female, excellent & $2.26 \%$ & $55.49 \%$ \\
\cline { 2 - 4 } & Male, good & $3.17 \%$ & $52.20 \%$ \\
\hline
\end{tabular}

\subsection{Verification of DEA-BP neural network model}

All the data are normalized so that the data of the input model are in the range of $[0,1]$. The processing equation is given as below:

$$
\left\{\begin{array}{c}
f: x \rightarrow y: y=\frac{x-x_{\min }}{x_{\max }-x_{\min }} \\
f: x \rightarrow y: y=\log _{10} x \\
f: x \rightarrow y: y=\frac{2 \arctan (x)}{\pi}
\end{array}\right.
$$

In the above equation, $x \max$ and xmin are the maximum and minimum of the data, respectively.

For the DEA-BP neural network model designed in this study, the variation factor is 0.6 , the population size is 25 , the crossover factor is 0.5 , the dimension is 6 , and the number of iterations is 10 . The basic structure of the BP neural network model is shown in Figure 10. The input layer contains a total of 4 neurons, and the output layer contains only 1 neuron. The number of neurons in hidden layer should be the optimal value during the model training. 


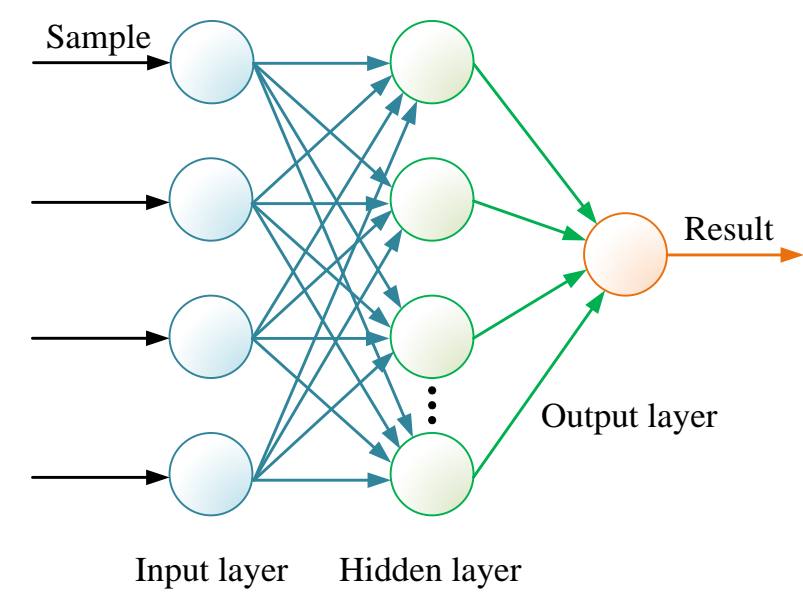

Fig. 10. Basic structure of the BP neural network model

Therefore, the learning rate is set to 0.1 , and the maximum training times is set to 1,000 in the BP neural network. The training errors when the number of hidden layer neurons is 1, 3, 5, 7, 9, and 11 are compared, respectively. Figure 11 illustrates that the training error is the smallest when the number of neurons in the hidden layer is 7; while the training error is the largest when the number of neurons in the hidden layer is 1 . Therefore, the number of neurons in the hidden layer is set to be 7 finally in this study for subsequent experiments.

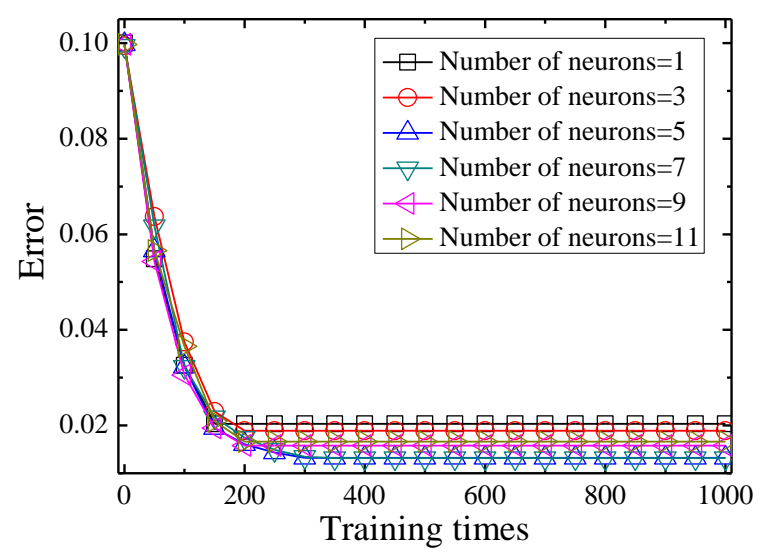

Fig. 11. Comparison on training errors of different numbers of neurons in the hidden layer

The differences in the training errors of models using SGD and Adam optimization algorithms, and the model using the Dropout and not using the Dropout are compared. 
Figure 12(a) and 12(b) show that the training errors of model using the Adam optimization algorithm and Dropout are smaller, which is similar to the research results of Mendenhall et al. (2016) [18]. Therefore, the Adam algorithm and the Dropout are used to optimize the DEA-BP neural network model finally in this study.

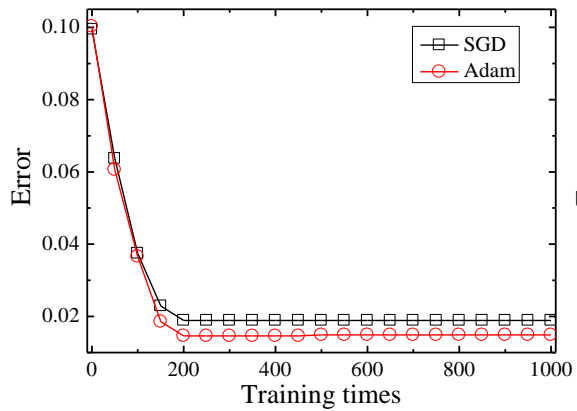

(a)

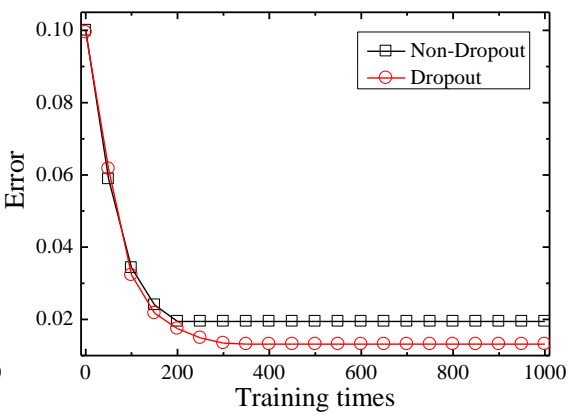

(b)

Fig. 12. Comparison on training errors of different optimization algorithms

The DEA-BP model is applied to the prediction of teacher's teaching evaluation results. The prediction error of DEA-BP model is compared with that of the Gradient Boosting Decision Tree (GBDT) algorithm [19] and the improved BP neural network model based on Particle Swarm Optimization (PSO-BP) algorithm [20]. The results are shown in Table 3. The average absolute error of the proposed DEA-BP neural network model is lower than that of the GBDT and PSO-BP, and its relative accuracy rate of predication is higher than that of the GBDT and PSO-BP. It suggests that applying the proposed DEA-BP algorithm to the teacher's teaching evaluation results can predict the final result better based on the rules of data.

Table 3. Comparison on predication of teaching evaulation results of different models

\begin{tabular}{|l|c|c|}
\hline \multicolumn{1}{|c|}{ Model } & Average absolute error (\%) & Relative accuracy rate (\%) \\
\hline GBDT & 1.42 & 93.19 \\
\hline PSO-BP & 3.20 & 90.52 \\
\hline DEA-BP & 1.05 & 95.44 \\
\hline
\end{tabular}

\section{Conclusion}

In this study, the potential correlation in the teaching evaluation data is mined based on the decision tree and modified Apriori algorithm. The results reveal that the teacher's age, gender, professional title, and academic qualification are all related to the final teaching evaluation results to some degree. The BP neural network algorithm improved by DEA can also realize the mining of potential correlation of the teaching evaluation data, and the accuracy rate of the final prediction of teaching evaluation results is higher. However, analysis is only made based on the existing teaching deci- 
sion-making system in this study, so an intelligent teaching evaluation decisionmaking system based on the related research results is necessary for in-depth analysis in future. In short, the results of this study can provide support for the mining of educational resource data and the intelligent development of teaching evaluation.

\section{Acknowledgement}

The work was supported by the National Natural Science Foundation of China (Grant: 111578109)

\section{$5 \quad$ References}

[1] Li, G., Hou, G., Wang, X., Yang, D., Hu, J. and Wang, W. (2018). A multivariate generalizability theory approach to college students' evaluation of teaching. Frontiers in Psychology, 9: 1065. https://doi.org/10.3389/fpsyg.2018.01065

[2] Heitkamp, S.J., Rüttermann, S. and Gerhardt-Szép, S. (2018). Work shadowing in dental teaching practices: evaluation results of a collaborative study between university and general dental practices. Bmc Medical Education, 18(1): 99. https://doi.org/10.1186/s12909$\underline{018-1220-4}$

[3] Keenan, P.B. and Jankowski, P. (2019). Spatial decision support systems: three decades on. Decision Support Systems, 116(JAN.): 64-76. https://doi.org/10.1016/j.dss.2018.10. $\underline{010}$

[4] Dali Luo. (2018). Using decision models to enable better irrigation decision support systems. International Journal of Emerging Technologies in Learning, 13(8): 90-102.

[5] Fanwen Kong. (2020). Application of Artificial Intelligence in Modern Art Teaching. Inter-national Journal of Emerging Technologies in Learning, 15(13): 238-251. https://doi.org/10.3991/ijet.v15i13.15351

[6] Cuibi Yang, Shuliang Huan, Yong Yang. (2020). A Practical Teaching Mode for Colleges Supported by Artificial Intelligence. International Journal of Emerging Technologies in Learning, 15(17): 195-206. https://doi.org/10.3991/ijet.v15i17.16737

[7] Ward, L., Dunn, A., Faghaninia, A., Zimmermann, N. E. R., Bajaj, S., Wang, Q., Montoya, J., Chen, J., Bystrom, K. and Dylla, M. (2018). Matminer: An open-source toolkit for materials data mining. Computational Materials Science, 152: 60-69. https://doi.org/10.1016/i.commatsci.2018.05.018

[8] Higashi, M., Ozaki, K., Hattori, T., Ishii, T., Soga, K., Sato, N., Tomita, M., Mizusawa, H., Ishikawa, K. and Yokota, T. (2018). A diagnostic decision tree for adult cerebellar ataxia based on pontine magnetic resonance imaging. Journal of the Neurological Sciences, 387: 187-195. https://doi.org/10.1016/j.jns.2018.02.022

[9] Attasena, V., Harbi, N. and Darmont, J. (2015). A novel multi-secret sharing approach for secure data warehousing and on-line analysis processing in the cloud. International Journal of Data Warehousing \& Mining, 11(2): 22-43. https://doi.org/10.4018/ijdwm.2015040102

[10] Charles, A. and Parks, G. (2019). Application of differential evolution algorithms to multiobjective optimization problems in mixed-oxide fuel assembly design. Annals of Nuclear Energy, 127: 165-177. https://doi.org/10.1016/j.anucene.2018.12.002

[11] Venturelli, O.S., Carr, A.C., Garth, F., Hsu, R.H., Rebecca, L., Bowen, B.P., Susan, H., Trent, N. and Arkin, A.P. (2018). Deciphering microbial interactions in synthetic human 
Paper-Intelligent Decision System of Higher Educational Resource Data Under Artificial Intelligence...

gut microbiome communities. Molecular Systems Biology, 14(6): e8157. https://doi.org/10.1101/228395

[12] Nguyen, B., Morell, C. and Baets, B.D. (2020). Scalable large-margin distance metric learning using stochastic gradient descent. IEEE Transactions on Cybernetics, 50(3): 10721083. https://doi.org/10.1109/TCYB.2018.2881417

[13] Khan, S. H., Hayat, M., Porkli, F. (2019). Regularization of deep neural networks with spectral dropout. Neural Networks, 110: 82-90. https://doi.org/10.1016/j.neunet.2018. $\underline{09.009}$

[14] Heng, J., Wang, J., Xiao, L. and Lu, H. (2017). Research and application of a combined model based on frequent pattern growth algorithm and multi-objective optimization for solar radiation forecasting. Applied Energy, 208(15): 845-866. https://doi.org/10.1016/ j.apenergy.2017.09.063

[15] Xia, X., Togneri, R., Sohel, F. and Huang, D. (2018). Random forest classification based acoustic event detection utilizing contextual-information and bottleneck features. Pattern Recognition, 81: 1-13. https://doi.org/10.1016/j.patcog.2018.03.025

[16] Reid and Landon, D. (2010). The role of perceived race and gender in the evaluation of col-lege teaching on RateMyProfessors.Com. Journal of Diversity in Higher Education, 3(3): 137-152. https://doi.org/10.1037/a0019865

[17] Kvach, E., Marcus, H. and Loomis, L. (2018). Evaluation of resident and faculty performance in routinely addressing unmet reproductive health needs in a teaching health center. Family Medicine, 50(4): 291-295. https://doi.org/10.22454/FamMed.2018.177339

[18] Mendenhall, J., and Meiler, J. (2016). Improving quantitative structure-activity relationship models using Artificial Neural Networks trained with dropout. Journal of computeraided molecular design, 30(2), 177-189. https://doi.org/10.1007/s10822-016-9895-2

[19] Wei, Z., Meng, Y., Zhang, W., Peng, J. and Meng, L. (2019). Downscaling SMAP soil moisture estimation with gradient boosting decision tree regression over the Tibetan Plateau. Remote Sensing of Environment, 225: 30-44. https://doi.org/10.1016/j.rse. $\underline{2019.02 .022}$

[20] Mei, Y., Yang, J., Lu, Y., Hao, F. and Wang, J. (2019). BP-ANN model coupled with particle swarm optimization for the efficient prediction of 2-chlorophenol removal in an electro-oxidation system. International Journal of Environmental Research and Public Health, 16(14): 2454. https://doi.org/10.3390/ijerph16142454

\section{$6 \quad$ Author}

Junhong Ma is a teacher of $\mathrm{Xi}$ 'an International University, associate professor of computer science, cloud computing development engineer. She has been engaged in the teaching and research of computer science and electronic information technology for a long time, research direction for Internet of Things and information processing.

Article submitted 2020-12-05. Resubmitted 2021-01-11. Final acceptance 2021-01-14. Final version published as submitted by the authors. 